\title{
Deep learning system for lymph node quantification and metastatic cancer identification from whole-slide pathology images
}

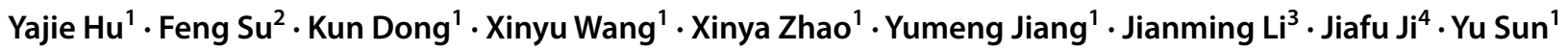

Received: 16 October 2020 / Accepted: 7 January 2021 / Published online: 23 January 2021

(C) The International Gastric Cancer Association and The Japanese Gastric Cancer Association 2021

\begin{abstract}
Background Traditional diagnosis methods for lymph node metastases are labor-intensive and time-consuming. As a result, diagnostic systems based on deep learning (DL) algorithms have become a hot topic. However, current research lacks testing with sufficient data to verify performance. The aim of this study was to develop and test a deep learning system capable of identifying lymph node metastases.

Methods 921 whole-slide images of lymph nodes were divided into two cohorts: training and testing. For lymph node quantification, we combined Faster RCNN and DeepLab as a cascade DL algorithm to detect regions of interest. For metastatic cancer identification, we fused Xception and DenseNet-121 models and extracted features. Prospective testing to verify the performance of the diagnostic system was performed using 327 unlabeled images. We further validated the proposed system using Positive Predictive Value (PPV) and Negative Predictive Value (NPV) criteria.

Results We developed a DL-based system capable of automated quantification and identification of metastatic lymph nodes. The accuracy of lymph node quantification was shown to be $97.13 \%$. The PPV of the combined Xception and DenseNet-121 model was $93.53 \%$, and the NPV was $97.99 \%$. Our experimental results show that the differentiation level of metastatic cancer affects the recognition performance.

Conclusions The diagnostic system we established reached a high level of efficiency and accuracy of lymph node diagnosis. This system could potentially be implemented into clinical workflow to assist pathologists in making a preliminary screening for lymph node metastases in gastric cancer patients.
\end{abstract}

Keywords Gastric cancer · Deep learning $\cdot$ Lymph node quantification $\cdot$ Lymph node metastasis

Yajie $\mathrm{Hu}$, Feng Su, Kun Dong and Xinyu Wang contributed equally to the study and are co-first authors.

Yu Sun

sunyu_bch@163.com

1 Key Laboratory of Carcinogenesis and Translational Research (Ministry of Education), Department of Pathology, Peking University Cancer Hospital and Institute, 52 Fucheng Road, Haidian District, Beijing 100142, China

2 Peking-Tsinghua Center for Life Sciences, Academy for Advanced Interdisciplinary Studies, Peking University, Beijing 100871, China

3 Institute for Artificial Intelligence, The State Key Laboratory of Intelligence Technology and Systems, Beijing National Research Center for Information Science and Technology, Department of Computer Science and Technology, Tsinghua University, Beijing 100084, China

4 Key Laboratory of Carcinogenesis and Translational Research (Ministry of Education), Gastrointestinal Cancer Center, Peking University Cancer Hospital and Institute, Beijing 100142, China

\section{Introduction}

Modeling of convolutional neural networks (CNN) has been successfully applied to the identification and classification of images for skin cancer, breast cancer, and other tumors $[1,2]$. Powered by rapid developments in digital histopathology, the performance of deep learning (DL) algorithms is on par with or even outperforms pathologists in visual identification [3]. Feng Su et al. established an automated diagnostic system of ascites pathology with $\mathrm{AUC}=0.8851$ and $96.80 \%$ precision [4]. Steiner et al. described the great potential of DL algorithms to make an accurate diagnosis of breast cancer [5]. Relative data show that the recognition of DL algorithms in the lymph node metastasis has reached $100 \%$ sensitivity [6]. Due to its innate capacity of extracting substantial attributes from 'big data', DL algorithms are expected to be the machine learning paradigm of medical diagnosis in the future [7]. 
The incidence of gastric cancer ranks high among malignant tumors worldwide [8]. For patients with advanced gastric cancer, pathological evaluation of the lymph node is critical to determine the stage of the disease, guide clinical treatments, and predict clinical prognosis [9]. The traditional diagnostic process for metastases is to use light microscopy to assess hematoxylin and eosin (HE)-stained sections. This enables pathologists to see whether tumor cells have invaded the lymph nodes and to count the number of metastatic lymph nodes [10]. This diagnostic procedure requires careful examinations of large-area tissues and is time-consuming. Moreover, there are uncontrollable subjective variabilities between the pathologists in terms of interpretation [11]. Therefore, DL-based diagnostic system not only significantly lightens the workload required to generate an accurate report, but also mitigates the subjective bias of pathologists [12, 13].

Owing to insufficient data, many statistics-driven studies require substantial efforts to conduct a prospective test of gastric cancer. By performing a prospective test with a wealth of data to assess its diagnostic performance, this study aimed to establish a DL-based diagnostic system to assist pathologists to make an auxiliary diagnosis of lymph node metastases of gastric cancer.

\section{Methods}

\section{Patient samples}

This study was a retrospective and diagnostic trial aiming to quantify the lymph nodes and identify the lymph node metastasis (LM). We obtained samples from the pathology archive at Beijing Cancer Hospital. This included 921 original hematoxylin and eosin (HE)-stained sections of 222 patients of gastric cancer (LM-positive, $n=176$; LMnegative, $n=46$, Tables 1,2$)$ who had undergone radical gastrectomy with lymph node dissection from 2009 to 2019 at Beijing Cancer Hospital. These samples were divided into training cohort and test cohort. Out of the 222 patients, 51 patients had undergone neoadjuvant therapy. The samples used in this study were all classified as lymph nodes either

Table 1 Patient data

\begin{tabular}{lrrl}
\hline & Whole & Train & Test \\
\hline Patients & 222 & 128 & 94 \\
Male & 170 & 108 & 62 \\
Female & 52 & 20 & 32 \\
Patients with LN metastases & 176 & 118 & 58 \\
Patients without LN metastases & 46 & 10 & 36 \\
Neoadjuvant chemotherapy & 51 & 38 & 13 \\
\hline
\end{tabular}

Table 2 Lymph node data

\begin{tabular}{lrrr}
\hline & Whole & Train & Test \\
\hline Images & 921 & 594 & 327 \\
Lymph nodes & 4004 & 2788 & 1216 \\
With metastases & 1323 & 1143 & 180 \\
$\quad$ Without metastases & 2681 & 1645 & 1036 \\
Histological type & & & \\
$\quad$ Adenocarcinoma & 1309 & 1130 & 179 \\
$\quad$ Neuroendocrine carcinoma & 14 & 13 & 1 \\
Differentiation & & & \\
$\quad$ Well differentiated & 123 & 45 & 78 \\
Poorly differentiated & 1200 & 1098 & 102 \\
Lauren classification & & & \\
Intestinal type & 120 & 101 & 19 \\
Diffuse type & 913 & 817 & 96 \\
Mixed type & 276 & 212 & 64 \\
\hline
\end{tabular}

with or without cancer metastasis. Slides without lymph nodes (such as fibrofatty tissue and cancer nodules) or with artifacts were removed. Ethical approval was granted from the institutional review board for this study. Sensitive information such as the patient's name, the medical record number, and ID number was removed from the files.

\section{Slide digitization}

The digitized whole-slide images (WSIs) were acquired at Beijing Cancer Hospital using a Leica Aperio Versa pathologic scanner (viewed at $400 \times$ magnification using Leica ImageScope software). The order of magnitude of pixels was $10^{9} \sim 10^{10}$.

\section{Architecture of the diagnostic system}

In this study, we developed a DL-based system to automate lymph node quantification and metastatic cancer identification from WSIs (Fig. 1a, b). The process of the proposed system was divided into two parts: lymph node quantification and gastric metastatic cancer identification. The proposed DL-based system was developed on a Linux platform with a P40 GPU with 24 GB memory.

\section{Annotation of the dataset}

Identification of individual lymph nodes was based on the nodular formation of lymphoid tissue and sinuses surrounded by a capsule of dense connective tissue. The diagnosis of lymph node metastasis was generally based on the following characteristics: total or focal lymph node involvement, significant necrosis, nest-like structure, and solid tumor plugs in the lymph node. 
a

Lymph node quantification

\begin{tabular}{|c|c|c|c|}
\hline $\begin{array}{l}\text { Whole-slide } \\
\text { pathological }\end{array}$ & $\begin{array}{l}\text { Preprocessing } \\
\text { (resize) }\end{array}$ & $\begin{array}{l}\text { Object detection } \\
\text { and segmentation }\end{array}$ & Lymph node \\
\hline (WSI) & $\begin{array}{l}\text { Preprocessing } \\
\text { (patches) }\end{array}$ & $\begin{array}{l}\text { Feature extraction } \\
\text { and classification }\end{array}$ & Metastatic cancer \\
\hline
\end{tabular}

Metastatic cancer identification

b

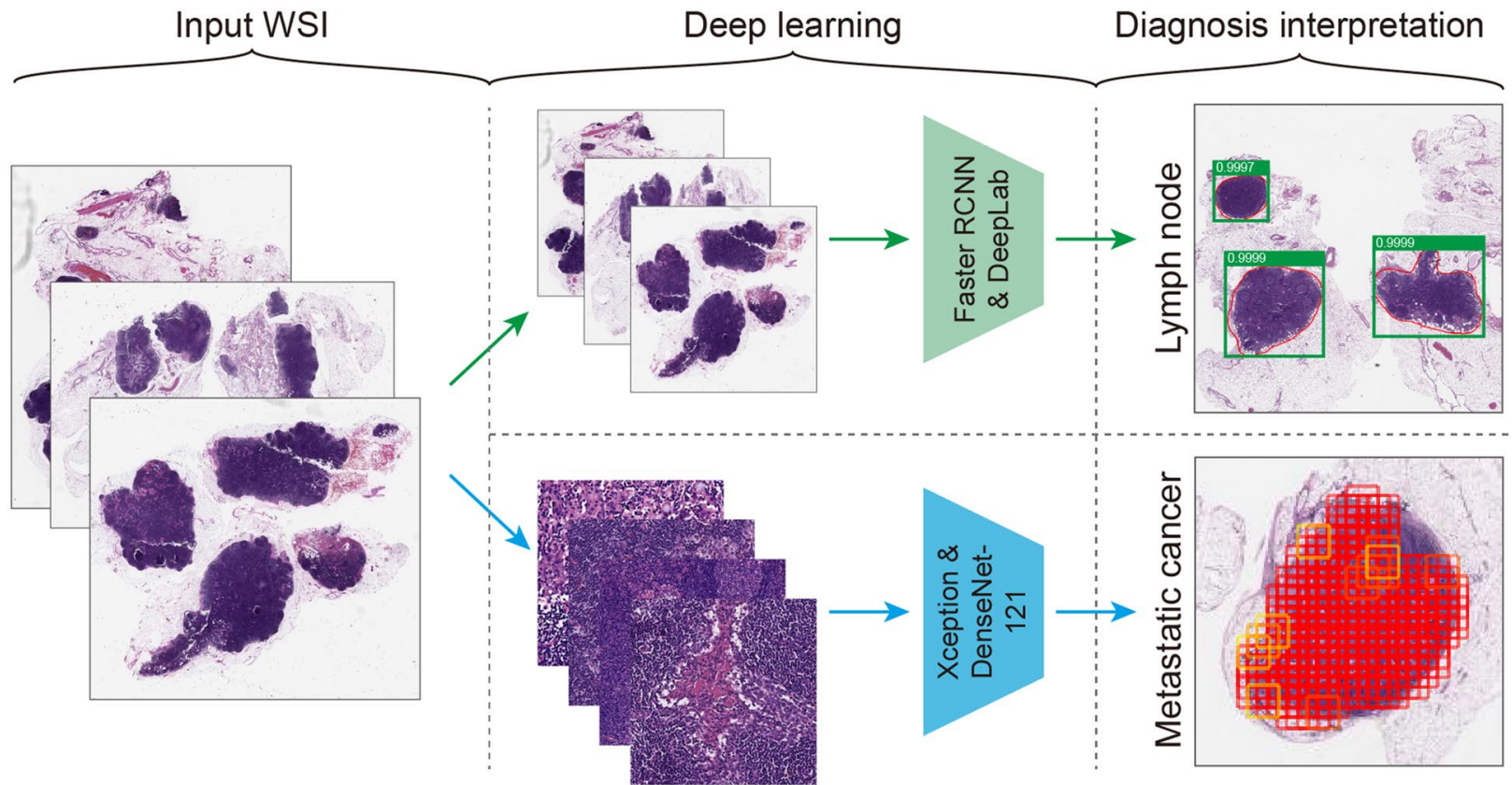

C

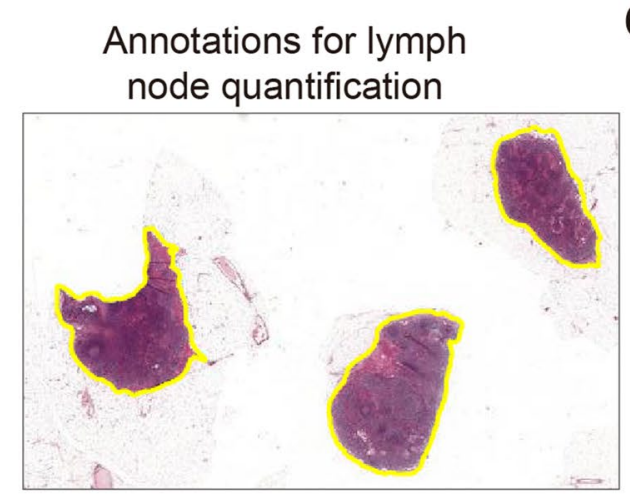

d

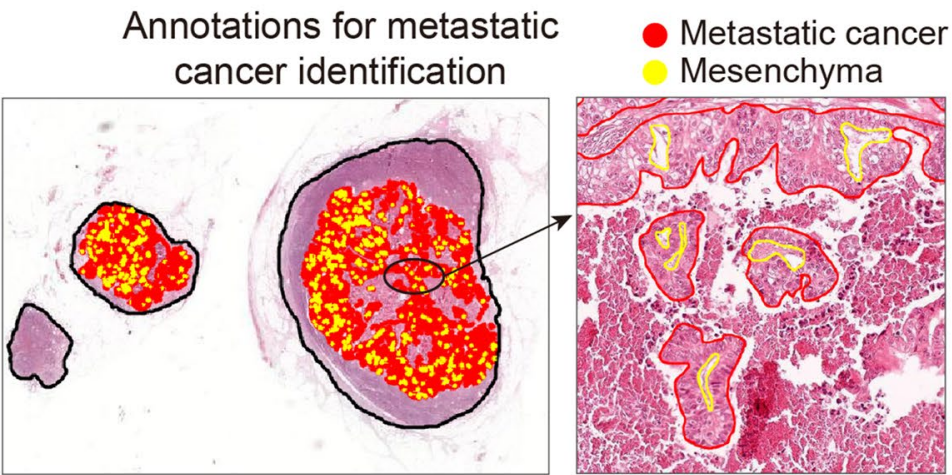

Fig. 1 Quantification and identification of metastatic lymph nodes by DL. a The architecture of DL-based system for lymph node and metastatic diagnosis. Green, the route for lymph node diagnosis. Blue, the route for metastatic cancer diagnosis. b An overview of the diagnosis process and results. The DL-based system received WSIs as input. The system used Faster RCNN and DeepLab models to diagnose lymph node after image normalization and outputted the contour and boundary box for each lymph node. The system used Xception and DensNet-121 models to diagnose metastatic cancer after patch extraction and outputted the risk value of each image patch for lymph node. c The annotations of WSI for lymph node quantification. The contours of all lymph nodes were outlined in yellow. $\mathbf{d}$ The annotations of WSI for metastatic cancer identification. Both the tumor (red) and the mesenchyma (yellow) were marked 
The pretreatment and annotations of WSIs were implemented under the supervision of a senior pathologist in an open precision medicine platform (AIMED, https://client.bloth ealth.com). Two experienced pathologists reviewed the digital images and marked the regions of interest (ROI). Where the lymph nodes had significant fibrosis, hemorrhage, or necrosis, pathologists labeled the cancer area and strictly avoided the fibrosis, hemorrhage, or necrosis. This reduced false-positive detection in samples as much as possible (Fig. 1c, d).

In the annotations for lymph node quantification, the region of the lymph node was outlined in yellow (Fig. 1c). In the annotations for metastatic cancer identification, both the tumor and the mesenchyma were denoted. The red line segments the organization of tumor infiltration and the yellow line marks the mesenchyma (Fig. 1d). When the results of the training cohort came out, a senior pathologist investigated and marked the areas of error detection as well as any undetected areas. Finally, the diagnostic system was updated with these new results.

\section{Dataset and models for lymph node quantification}

We combined Faster RCNN [14], which is a state-of-art DL algorithm for object detection, and DeepLab [15], which is a state-of-the-art DL model for semantic image segmentation as a cascade DL system. Resnet-50 [16], which has won many competitions such as ILSVRC and COCO 201, was selected as the feature extractor for Faster RCNN. In brief, we trained a segmentation model on WSIs of digitized pathology slides with the cascade DL algorithm. WSIs with pixels between $2000 \times 2000$ and $3000 \times 3000$ were resized with an appropriate scale factor.

The images were randomly divided into training dataset (594 annotated images) and testing dataset (327 unlabeled images). In the training stage, an object detection model was trained to detect the ROIs corresponding to the lymph nodes on the whole image. Then a segmentation model was trained to dissect the lymph nodes within the ROI. Finally, we combined the results from two models to achieve full quantification of the lymph nodes (Fig. 2a, b). The transfer learning technique was used to train the models. This was performed by fine-tuning the weights of the DL models to reduce the difference between the predicted output and the annotation. Finally, the number of lymph nodes was determined on the WSI according to the threshold of the likelihood. This threshold was set to 0.5 (Fig. 2c). The models were trained 346 epochs. The initial learning rate was 0.01 .

\section{Dataset and models for metastatic cancer identification}

Due to the large size of the WSI, direct processing of the original image requires huge levels of computer memory.
To overcome this issue, we generated a mask image for the WSI according to annotations. The initial value of the mask was $(255,255,255)$ and it was the same size as the WSI. The values of the mask image corresponding to the marked area were set as $(0,0,0)$. Second, we extracted multiple image patches from WSI. This was performed using the mask image. The size of the image patch was $512 \times 512$ and the stride was 256 . The image patch extraction operation started from the upper left corner of the area with the pixel value of $(0,0,0)$ on the mask until all the area with the pixel value of $(0,0,0)$ in the mask image was covered.

In this study, two models, Xception and DenseNet-121, were combined to achieve metastatic cancer identification. Xception and DenseNet-121 enhanced the network depth and greatly reduced the number of parameters. The two networks were used to extract features, and each network was connected to global maximum pooling. The pooled features were concatenated, followed by the full connection layer for classification. 921 images of 4004 lymph nodes (LMpositive, $n=1323$; LM-negative, $n=2681$ ) were divided into training cohort (594) and test cohort (327). We adopted Adam optimization method to train the model. The initial learning rate was 0.002 and the batch size was 16 .

\section{Prospective cohort of the diagnostic system}

After fine-tuning the algorithm with repeated checking and optimization, the diagnostic system was established. To fully evaluate the performance, we incorporated 327 images as the prospective test. As illustrated in Tables 1 and 2, we enrolled 94 patients (LM-positive, $n=58$; LM-negative, $n=36$ ) who had undergone radical gastrectomy with lymph node dissection at Beijing Cancer Hospital.

\section{Results}

\section{Quantitative evaluation of lymph node quantification}

First, the WSI pathology images were preprocessed and normalized according to the parameter settings of the DL model (Fig. 1a, b). Next, we used transfer learning techniques to fine-tune the parameters of pre-trained Faster RCNN and DeepLab models to achieve lymph node detection and segmentation. We utilized a DeepLab model after the Faster RCNN model to improve the accuracy of lymph node quantification (Fig. 2a).

First, Faster RCNN made an initial detection of the lymph nodes in the pathological image. The boundary box obtained by Faster RCNN detection could contain multiple lymph nodes. Then the detection results of Faster RCNN were fed into the DeepLab model for further segmentation. This 
a

b
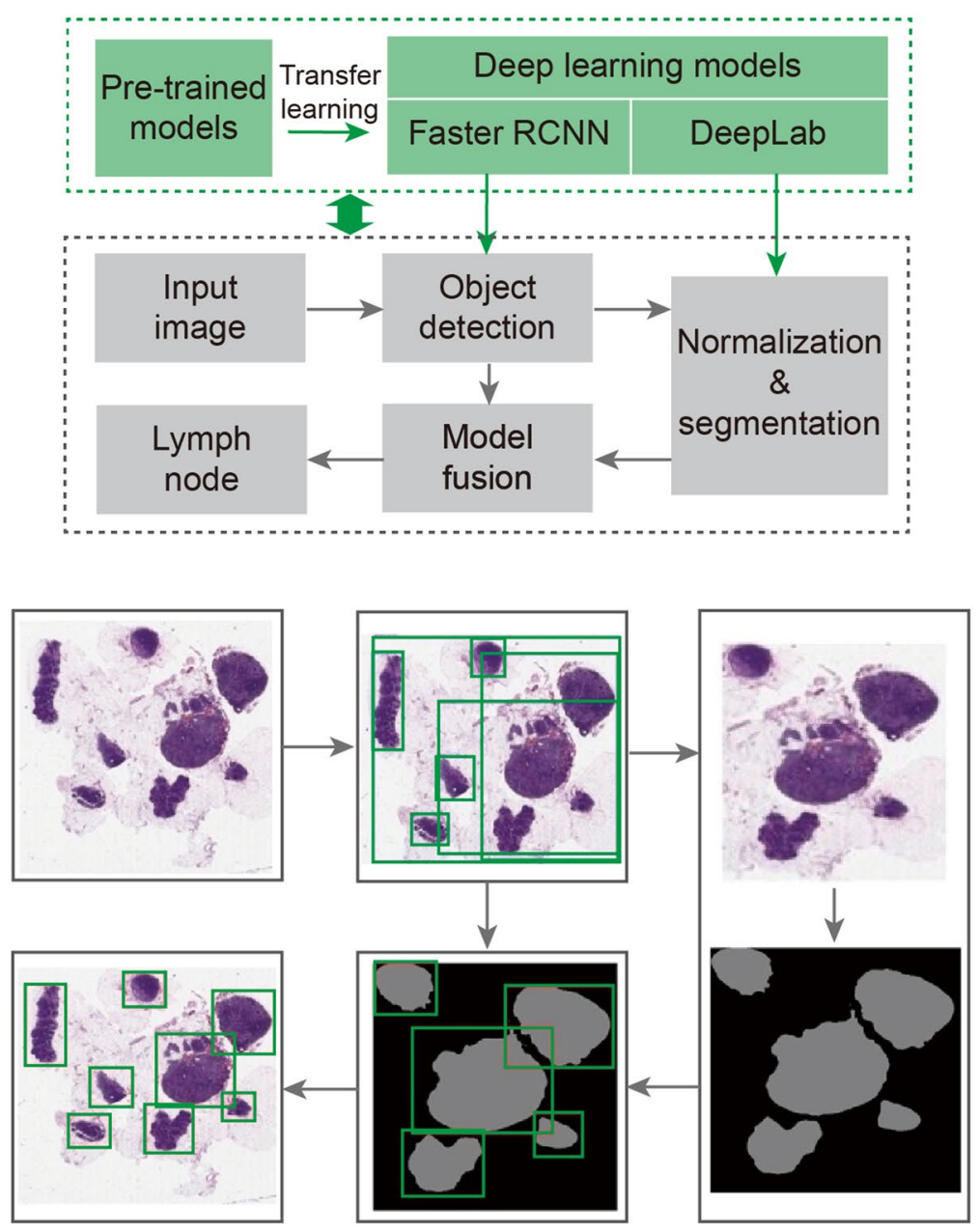

C
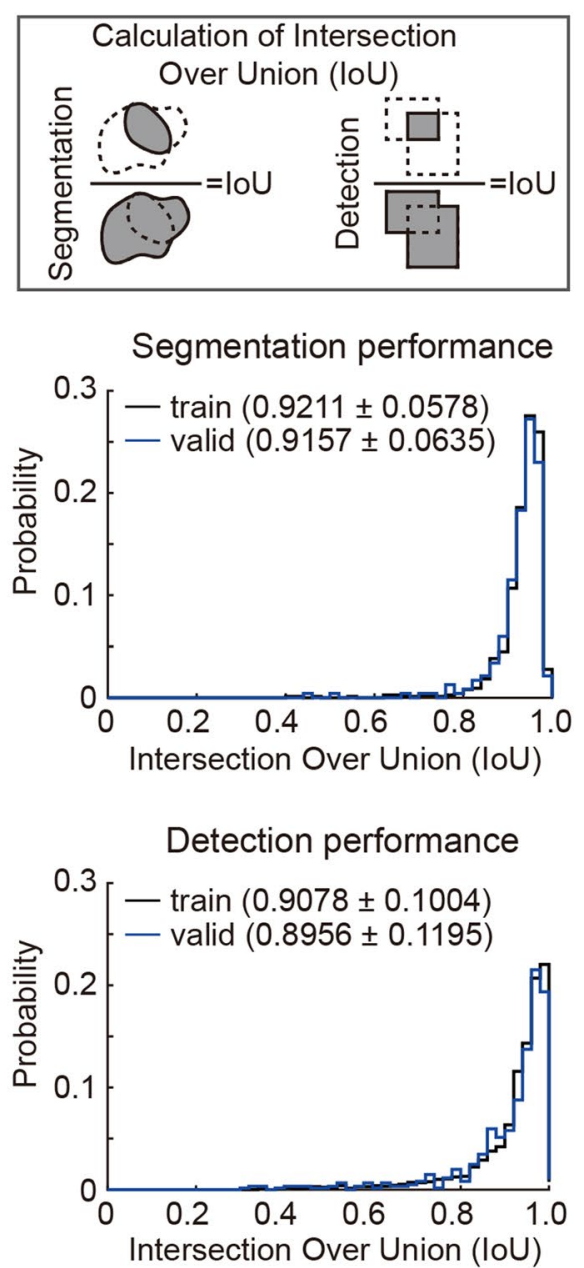

Fig. 2 Lymph node diagnosis by DL-based system. a The architecture of the proposed system for lymph node diagnosis. The system combined Faster RCNN and DeepLab as a cascade DL algorithm to detect the lymph node. $\mathbf{b}$ An overview of the lymph node diagnosis. The system used Faster RCNN model to detect lymph node and output boundary box first. The detection results were further refined by DeepLab model and segmented the contour of each lymph node. The system fused the results from Faster RCNN and DeepLab to output

facilitated accurate lymph node segmentation. Finally, the results from the Faster RCNN and DeepLab models were fused to enable the successful lymph node quantification (Fig. 2b).

For both target segmentation and detection, Intersection over Union (IoU) metrics were used to evaluate the performance of the system. IoU characterized the overlap between the annotation and the results of models. In the lymph node segmentation task, the DeepLab model achieved excellent performance with regard to both training and testing procedures (mean \pm std; training, $0.9211 \pm 0.0578$; testing, $0.9157 \pm 0.0635)$. In terms of lymph node detection, the model-fused DL system also achieved high IoU (mean \pm std; training, $0.9078 \pm 0.1004$; testing, $0.8956 \pm 0.1195$ ). To diagnosis results. $\mathbf{c}$ The results of lymph node diagnosis performance. Upper, criterion of Intersection over Union (IoU) to evaluate the performance of lymph node detection and segmentation. Middle, histogram of IoU for lymph node segmentation by DeepLab in the training and validation process. Lower, histogram of IoU for lymph node detection by fused Faster RCNN and DeepLab models in the training and validation process

achieve lymph node quantification, a threshold value of 0.5 was set for IoU. During validation, the system achieved a lymph node technical accuracy of $97.13 \%$ (Fig. 2c).

To achieve lymph node quantification, we combined the techniques of object detection and object segmentation based on deep CNN. The goal of this task was to obtain the boundary box of the lymph node, and the goal of the object segmentation task was to obtain the contour of the lymph node. There were some non-lymph node areas in the boundary box obtained by object detection. In IoU-based assessment metrics, the successful performance of the object detection task was dependent on several factors, including the location and morphology of the lymph nodes. When the lymph nodes were well separated and their respective 
a

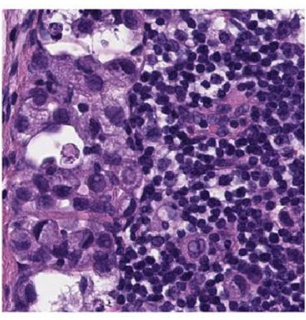

Image patch
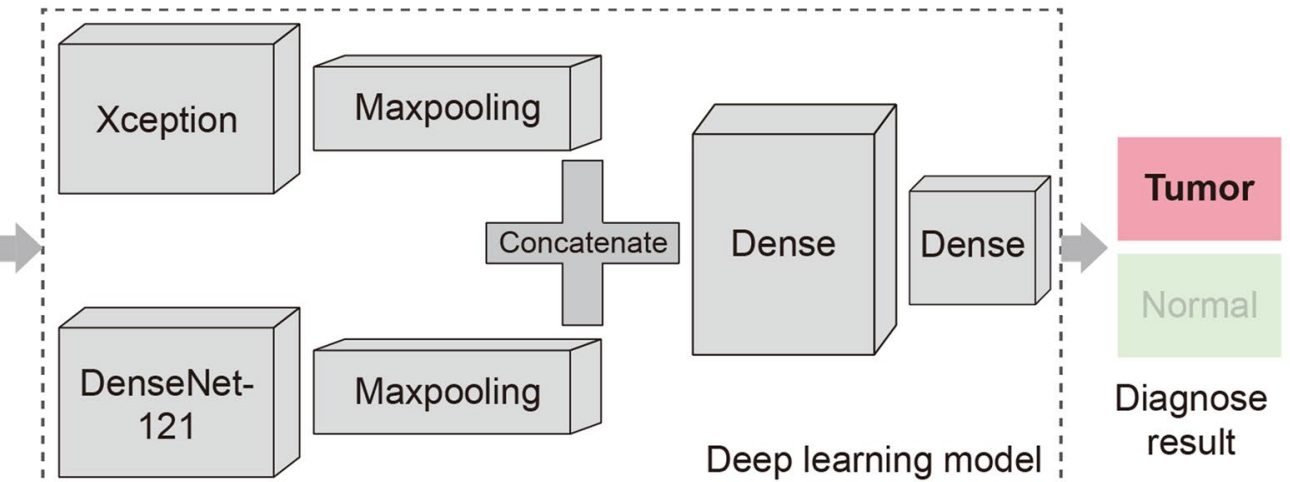

Diagnose

Deep learning model

b

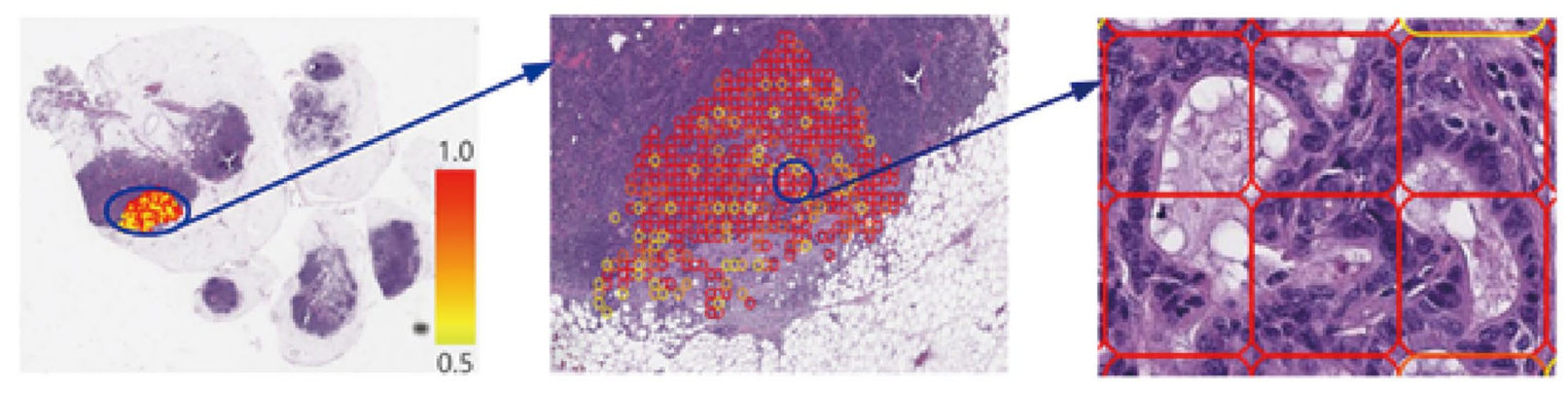

C

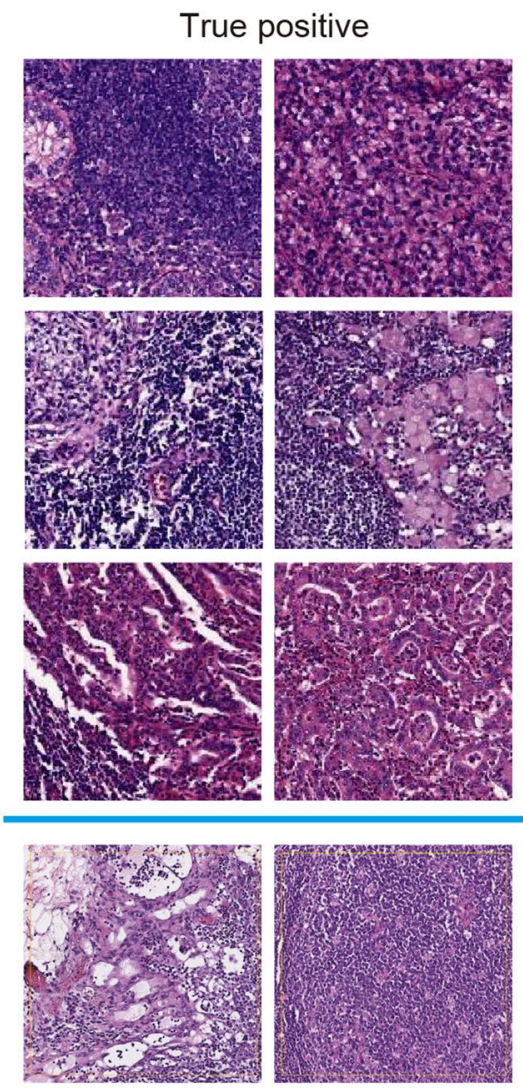

False positive
False negative
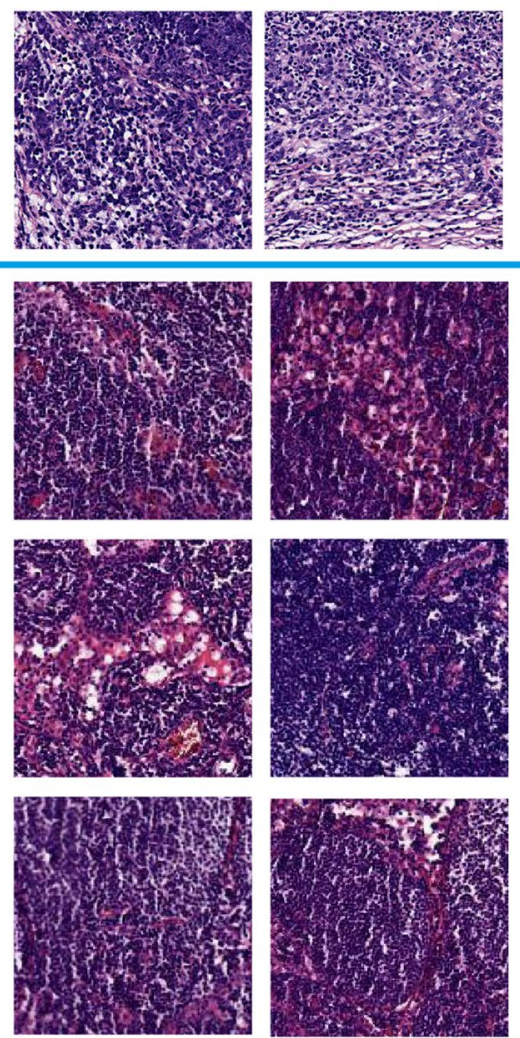

True negative
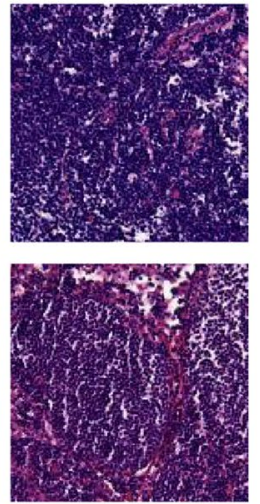

d

Performance summary (Xception \& DenseNet-121)

\begin{tabular}{|c|c|c|c|}
\hline \multirow{3}{*}{ 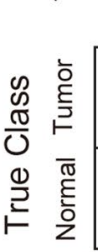 } & \multicolumn{2}{|c|}{ Samples } & Ratio \\
\hline & 159 & 21 & $\begin{array}{ll}\stackrel{\circ}{\circ} & \stackrel{0}{\circ} \\
\infty & \stackrel{\circ}{\risingdotseq}\end{array}$ \\
\hline & 11 & 1025 & $\begin{array}{l}\circ \\
\circ \\
\infty \\
\infty\end{array}$ \\
\hline & $93.5 \%$ & $98.0 \%$ & \\
\hline & $6.5 \%$ & $2.0 \%$ & \\
\hline
\end{tabular}

e

Comparison between three models

\section{口 DenseNet-121}

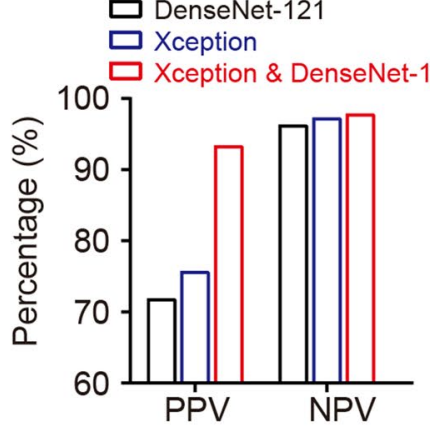


4Fig. 3 Metastatic cancer diagnosis by DL-based system. a The architecture of the proposed system for metastatic cancer diagnosis. The system combined Xception and DenseNet-121 models to diagnose the metastatic cancer. The features extracted by Xception and DenseNet-121 models were concatenated to increase the performance of the proposed system. A positive image patch sample was classified as tumor by the system. b An overview of the diagnosis process and results. The system calculated the risk value of metastatic cancer for each image patch of lymph node. c Image patch samples of true-positive (TP), false-positive (FP), false-negative (FN), and truenegative $(\mathrm{TN})$ classification by the proposed system. d The predicted performance of Xception \&DenseNet-121 in the validation cohort. $\mathrm{e}$ A comparison between three models (DenseNet-121, Xception, and Xception \& DenseNet-121)

envelops were clear, the accuracy of lymph node quantification was high. But in cases where lymph nodes were closer and separation was more difficult (even there was only a little structural connection between lymph nodes), the boundary boxes crossed over each other. This led to decreased accuracy. Nevertheless, the Faster RCNN model played a very important role in the lymph node quantification task and was able to locate the lymph node regions quickly and efficiently. It should be noted that cascading an object segmentation model, such as DeepLab, after the Faster RCNN for further fine-grained segmentation of lymph nodes can adequately address the shortcomings of object detection algorithms. Object segmentation models are generally more complex and are difficult to be trained directly on the original WSIs. The DL-based system we proposed in this study combined the advantages of Faster RCNN and DeepLab models. The proposed system had high accuracy in lymph node quantification.

\section{Quantitative evaluation for the identification of metastatic cancer}

WSI images were too large to be directly inputted into the model for metastatic cancer identification. What's more, in many cases, there were background areas in the images that did not need analysis. Doing so would substantially increase the consumption time to run the model but with no benefit to the accuracy. In this study, we extracted standardized image patches from the WSI. Then we inputted the image patches to the model for tumor identification (Fig. 1a, b). In our established deep learning system, a model consisting of a combination of Xception and DenseNet-121 models was used. Xception and DenseNet-121 extracted features from the input image blocks and fused them by concatenating to obtain the final recognition results (Fig. 3a). The DL-based system performed intelligent metastatic cancer identification on WSI. The system produced tumor identification results as a heat map (Fig. 3b). We observed several samples that were true-positive, false-positive, true-negative, and falsenegative image patches for the DL-based system (Fig. 3c).
Our experimental results showed that the proposed system with fused DL models was extremely capable of tumor recognition (Fig. 3d).

To test the diagnostic performance of the three models (Xception, DenseNet, combined Xception and DenseNet), we, respectively, calculated their positive and negative predictive values. The positive predictive value (PPV) denoted the ratio of true positive samples over the prediction-positive samples. The negative predictive value (NPV) denoted the ratio of true negative samples over the prediction-negative samples. The PPV and NPV of single DenseNet-121 were $72 \%$ and $96.46 \%$, respectively. The PPV and NPV of single Xception were $75.86 \%$ and $97.44 \%$, respectively. The PPV and NPV of combined Xception and DenseNet-121 were $93.53 \%$ and $97.99 \%$, respectively (Fig. 3e). Excitingly, the combined Xception and DenseNet-121 achieved the optimal metastatic cancer diagnostic level.

\section{Cause of error and omission}

The proposed system correctly classified most of the image patches. However, there were several false positive and negative samples (Fig. 3c). We further analyzed the relationship between the characteristics of lymph nodes and the performance of DL-based systems.

Positive lymph nodes: according to the results of the prospective test, we suggest that the accuracy of identifying metastatic lymph nodes is closely related to the differentiation degree of metastatic carcinoma. By analyzing the sample information, the differentiation of metastases in lymph nodes was studied in great detail. Among the prospective test results, there were 180 positive lymph nodes and 1036 negative lymph nodes. Of which, $43.33 \%(N=78)$ were moderately differentiated adenocarcinoma, $86(47.78 \%)$ were poorly differentiated adenocarcinoma, $8.33 \%(N=15)$ were signet ring cell carcinoma, and $0.56 \%(N=1)$ were neuroendocrine carcinoma. The model predicted that the False Negative Rate (FNR) was $15.11 \%$ for poorly differentiated adenocarcinoma, and $2.56 \%$ for moderately differentiated adenocarcinoma. There were no pseudo-positive lymph nodes for either group. The FNR of a signet ring cell carcinoma was $6.67 \%$ and the false-positive rate (FPV) was $0.10 \%$. Lymph node metastases from neuroendocrine carcinoma were identified correctly. Due to the different differentiation and pathological characteristics of each type of metastatic carcinoma, the identification efficiency of the model was markedly different. Grade 1 (G1): well-differentiated adenocarcinoma (more than $95 \%$ of tumor cells form adenocarcinoma ducts); G2: moderately differentiated adenocarcinoma (50-95\% of tumor cells form adenocarcinoma ducts); G3: poorly differentiated adenocarcinoma (adenotubules form in tumor cells up to $49 \%$ of the time). The adenocarcinoma ducts were well differentiated with a clear boundary 
and so the model recognition efficiency was relatively high. However, in adenocarcinoma with poor differentiation, there are few glandular tubules, unclear boundaries, and extensive infiltration growth. In these cases, the model recognition efficiency was low. Moreover, in signet ring cell carcinoma, which is characterized by signet-ring-like cells, it was found that the recognition performance is closely related to the number of cancer cells. When there are a large number of signet ring cells, in a concentrated space, the model is able to accurately identify them. However, when the cells are few and scattered, the model is prone to misdiagnosis. For some poorly differentiated carcinoma, it is difficult to distinguish them by HE alone. In these cases, immunohistochemistry (IHC) was used to further verify diagnosis.

Negative lymph nodes: The TNR (true negative rate) was $98.94 \%$ and the FPR was $1.06 \%$. The germinal center of lymph nodes is easily misdiagnosed as metastatic tumorcell lumps, as there are cell division and proliferation in the center.

One potential limitation to this study is that it is single center. To ensure wider relevance of this system and our study, we propose a multi-center collaboration in the next stage to improve the applicability. Furthermore, there are several other factors such as the architecture of the DL model and the balance of the training data would affect the performance of the diagnostic system. We hypothesize that with wider validation from other centers, this diagnostic system has potential utility in the clinical diagnostic workflow.

\section{Validation of the DL system by neoadjuvant chemotherapy}

Currently, neoadjuvant chemotherapy (NACT) is used to reduce cancer foci, improve surgical resection rate, and improve long-term prognosis in patients with advanced gastric cancer. The lymph nodes of postoperative patients will also produce a post-treatment response, such as metastatic tumor shrinkage and fibrosis, etc. To test whether the model was suitable for the identification of lymph nodes after NACT, during model construction, we randomly added lymph node images from 38 patients who underwent NACT (33 of whom had lymph node metastasis) as training data. We then used lymph nodes from 13 patients after NACT to validate the diagnostic model. Among the 13 patients treated with NACT, there were 218 lymph nodes and 18 of them were positive lymph nodes. Among these metastatic carcinoma types included $55.56 \%(N=10)$ poorly differentiated adenocarcinoma, 33.33\% $(N=6)$ moderately differentiated adenocarcinoma, $5.56 \%(N=1)$ signet ring cell carcinoma, and $5.56 \%(N=1)$ neuroendocrine carcinoma. After model diagnosis, the positive predictive value was $94.44 \%$ and the negative predictive value was $99.5 \%$. The results show that the model is effective and can be used for lymph node screening after NACT with confidence.

\section{Discussion}

The lymph node is the most common site of malignant tumor metastasis. In many cases, the lymph node is the first clinical manifestation of the disease. The number of metastatic lymph nodes is a decisive index of the tumor node metastasis (TNM) stage of gastric cancer. TNM is considered to be one of the most pivotal factors in evaluating the prognosis of gastric cancer [9]. An important role of the pathologist is to determine whether there are metastases in the lymph nodes and to quantify them.

In this study, we developed and validated an intelligent DL-based system for pathological images from gastric cancer pathology. This system achieved automated lymph node quantification and metastatic cancer identification. This system is an end-to-end system capable of directly analyzing WSIs. In comparison to systems that can only handle preprocessed crops of WSI, the analysis of WSIs has the potential to reduce the probability of misdiagnosis. As such it is easier to integrate into the clinical diagnostic process [4]. What's more, the proposed DL-based system employs a model fusion technique, which can improve the reliability and accuracy of diagnosis. The combination of Faster RCNN for accurate and efficient lymph node detection [17] and DeepLab for precise lymph node segmentation [18] offers the potential for efficient lymph node quantification. Faster RCNN is one of the state-of-the-art object detection models that enable the fast detection of targets in a large field of view. However, one of the limitations of Faster RCNN lies in that it only supports rectangular annotations, which has poor performance in the case of small object size and complex contours. To address these limitations, we use the cascaded DeepLab model to achieve fine contour segmentation of individual lymph nodes, which can effectively improve the accuracy of lymph node quantification. Besides, the combination of Faster RCNN and DeepLab algorithms was also proved efficient in other medical applications such as liver segmentation [19]. This study presents a robust system, which boasts the advantage of including multiple models. To facilitate cancer metastasis, we use fused Xception and DenseNet-121 models to extract image features. The reliability of Xception and DenseNet-121 as individual models [20] has been validated in several medical image studies [21]. However, DL models are usually biased in feature extraction because of the model architecture and training methods. It is also important to note that the systems based on a single model have limitations in dealing with complex tasks. In practical applications, the fusion of multiple DL models is widely used to improve the accuracy and stability of 
systems, including image processing [22] and robot control [23]. AI-related diagnosis and treatment methods provide more technical support for precision medicine. For instance, AI-based medical device of diabetic retinopathy detection has been permitted marketing by the Food and Drug Administration (FDA) [24], which, therefore, reduces the risk of unnecessary tests and increases the precision medical rate. Just as Naylor identifies [25], there are some unique advantages to drive AI and deep learning applied to health, such as the superior performance of digital image analyzation, more convenient sharing of medical data, more broadly applicable to heterogeneous medical data, the potential to streamline clinical workflows and provide convenience to patients, and the open-source deep learning programs.

In this study, we fused the features extracted from the Xception and DenseNet-121 models and demonstrated that the fused model achieved the highest accuracy in metastasis cancer identification. Finally, the proposed DL-based system was open and capable of continuous evolution. For new datasets, the system was able to quickly achieve diagnosis by accepting evaluation and correction from experts. This means the system can be re-trained to achieve continuous optimization.

Acknowledgements This work was supported by the National Key Research and Development Program of China (2018YFC0910700), the Major Program of National Natural Science Foundation of China (91959205), and Capital's Funds for Health Improvement and Research, CFH, No. 2018-2Z-1026.

Author contributions Conception and design: YS and JJ; development and methodology: YH, FS, and JL; acquisition of data (acquired and managed patients, provided facilities, etc.): $\mathrm{YH}, \mathrm{KD}, \mathrm{XW}$, and $\mathrm{YJ}$; analysis and interpretation of data (e.g., statistical analysis, computational analysis): YH, FS, KD, XW, XZ, JL, JJ, and YS; writing, review, and/or revision of the manuscript: YH, FS, KD, XW, XZ, JL, JJ, and YS; administrative, technical, or material support (i.e., reporting or organizing data, constructing databases): YS, JJ, and JL.

\section{Compliance with ethical standards}

Conflict of interest The authors declare no potential conflicts of interest.

\section{References}

1. Golden JA. Deep learning algorithms for detection of lymph node metastases from breast cancer: helping artificial intelligence be seen. JAMA. 2017;318(22):2184-6. https://doi.org/10.1001/ JAMA.2017.14580.

2. Esteva A, Kuprel B, Novoa RA, Ko JM, Swetter SM, Blau HM, et al. Dermatologist-level classification of skin cancer with deep neural networks. Nature. 2017;542(7639):115-8. https://doi. org/10.1038/NATURE21056.

3. Russakovsky O, Deng J, Su H, Krause J, Satheesh S, Ma S, et al. ImageNet Large scale visual recognition challenge. Int J
Comput Vision. 2015;115(3):211-52. https://doi.org/10.1007/ S11263-015-0816-Y.

4. Su F, Sun Y, Hu Y, Yuan P, Wang X, Wang Q, et al. Development and validation of a deep learning system for ascites cytopathology interpretation. Gastric Cancer. 2020. https://doi. org/10.1007/S10120-020-01093-1.

5. Steiner DF, MacDonald R, Liu Y, Truszkowski P, Hipp JD, Gammage $C$, et al. Impact of deep learning assistance on the histopathologic review of lymph nodes for metastatic breast cancer. Am J Surg Pathol. 2018;42(12):1636-46. https://doi. org/10.1097/PAS.0000000000001151.

6. Litjens G, Sánchez CI, Timofeeva N, Hermsen M, Nagtegaal I, Kovacs I, et al. Deep learning as a tool for increased accuracy and efficiency of histopathological diagnosis. Sci Rep. 2016;6(1):26286. https://doi.org/10.1038/SREP26286.

7. Janowczyk A, Madabhushi A. Deep learning for digital pathology image analysis: a comprehensive tutorial with selected use cases. J Pathol Inform. 2016;7(1):29. https://doi. org/10.4103/2153-3539.186902.

8. Song Z, Zou S, Zhou W, Huang Y, Shao L, Yuan J, et al. Clinically applicable histopathological diagnosis system for gastric cancer detection using deep learning. Nature Commun. 2020. https://doi.org/10.1038/S41467-020-18147-8.

9. Zhao B, Huang R, Lu H, Mei D, Bao S, Xu H, et al. Risk of lymph node metastasis and prognostic outcome in early gastric cancer patients with mixed histologic type. Current Problems Cancer. 2020. https://doi.org/10.1016/J.CURRPROBLCANCER .2020 .100579$.

10. Sun F, Liu S, Song P, Zhang C, Liu Z, Guan W, et al. Impact of retrieved lymph node count on short-term complications in patients with gastric cancer. World J Surg Oncol. 2020;18(1):17. https://doi.org/10.1186/S12957-020-02000-9.

11. Shubhasini AR, Praveen BN, Usha Hegde UK, Shubha $\mathrm{G}$, Keerthi $\mathrm{G}$, et al. Inter- and intra-observer variability in diagnosis of oral dysplasia. Asian Pacific J Cancer Prevention. 2017;18(12):3251-4. https://doi.org/10.22034/APJCP .2017.18.12.3251.

12. Bhargava R, Madabhushi A. Emerging themes in image informatics and molecular analysis for digital pathology. Ann Rev Biomed Eng. 2016;18(1):387-412. https://doi.org/10.1146/ANNUREVBIOENG-112415-114722.

13. Veta M, Pluim JPW, Diest PJ, Viergever MA. Breast cancer histopathology image analysis: a review. IEEE Trans Biomed Eng. 2014;61(5):1400-11. https://doi.org/10.1109/TBME.2014.23038 52.

14. Ren S, He K, Girshick R, Sun J. Faster R-CNN: towards real-time object detection with region proposal networks. IEEE Trans Pattern Anal Machine Intelligence. 2017;39(6):1137-49. https://doi. org/10.1109/TPAMI.2016.2577031.

15. Zhao H, Shi J, Qi X, Wang X, Jia J. Pyramid scene parsing network. Computer Vision and Pattern Recognition; 7/21/20172017. p. 6230-9

16. Targ S, Almeida D, Lyman K. Resnet in Resnet: generalizing residual architectures. 2016.

17. Ding L, Liu G-W, Zhao B-C, Zhou Y-P, Li S, Zhang Z-D, et al. Artificial intelligence system of faster region-based convolutional neural network surpassing senior radiologists in evaluation of metastatic lymph nodes of rectal cancer. Chin Med J. 2019;132(4):379-87. https://doi.org/10.1097/CM9.0000000000 000095.

18. Pan Y, Sun Z, Wang W, Yang Z, Jia J, Feng X, et al. Automatic detection of squamous cell carcinoma metastasis in esophageal lymph nodes using semantic segmentation. Clin Trans Med. 2020. https://doi.org/10.1002/CTM2.129.

19. Tang W, Zou D, Yang S, Shi J, Song GJNC. Applications. A two-stage approach for automatic liver segmentation with Faster 
R-CNN and DeepLab. Neural Comput Appl. 2020. https://doi. org/10.1007/s00521-019-04700-0.

20. Ardakani AA, Kanafi AR, Acharya UR, Khadem N, Mohammadi A. Application of deep learning technique to manage COVID-19 in routine clinical practice using CT images: results of 10 convolutional neural networks. Comput Biol Med. 2020. https://doi. org/10.1016/J.COMPBIOMED.2020.103795.

21. Minaee S, Kafieh R, Sonka M, Yazdani S, Soufi GJ. Deep-COVID: predicting COVID-19 from chest X-ray images using deep transfer learning. Med Image Anal. 2020. https://doi.org/10.1016/J. MEDIA.2020.101794.

22. Deng Z, Zhu L, Hu X, Fu CW, Heng PA, editors. Deep multimodel fusion for single-image Dehazing. International Conference on Computer Vision (ICCV), 2019; 2019.

23. Wang T, Zhang H, Toh WQ, Zhu H, Jing W, editors. Efficient Robotic Task Generalization Using Deep Model Fusion
Reinforcement Learning. 2019 IEEE International Conference on Robotics and Biomimetics (ROBIO); 2019.

24. Sampson DK, Dwyer LM, Tseng EH, Lorell BH, Kavi KJ, FDA proposes regulatory framework for artificial intelligence/machine learning software as a medical device. J tl 2019;31(7):12-6.

25. Naylor $\mathrm{CD}$. On the prospects for a (deep) learning health care system [published online August 30, 2018]. JAMA. 2018. https ://doi.org/10.1001/jama.2018.11103.(1).

Publisher's Note Springer Nature remains neutral with regard to jurisdictional claims in published maps and institutional affiliations. 